Sheyla Moroni

Università degli Studi di Firenze

\title{
El 2000 Visto Desde El PeOPle's Party
}

\section{The Year 2000 from the Perspective of the People's Party}

\begin{abstract}
For a few years (1890-1896), the People's Party was the "third party" of the United States and it changed the rhetoric of the two major parties into which it "dissolved" (especially in the case of the Democratic Party represented by William Jennings Bryan in 1896). The aim of the article is to test the politicalnarrative background which includes, among other productions in 1888, the first edition of Looking Backward. 2000-1887 by Edward Bellamy. One million copies of this book were sold and its readers created half a thousand nationalist clubs in the US. Caesar's Column by Ignatius L. Donnelly's (1890), author of the Preamble of the Party, was also widely sold.
\end{abstract}

Keywords: People's Party, populism, history of the United States, dystopia, utopia

\section{Resumen}

El People's Party ha sido para algunos el "tercer partido" de los Estados Unidos (1890-1896), el cual cambió la retórica de los dos partidos principales en los que se "disolvió", de forma más evidente en el caso del Partido Democrático, representado por William Jennings Bryan en 1896. El artículo se propone evaluar el background político-narrativo que cuenta, entre otras producciones en 1888, con la primera edición de Looking Backward. 2000-1887 de Edward Bellamy, la cual vendió un millón de copias y suscitó el nacimiento de medio millar de sociedades de tipo nacionalista en los USA, así como lo hizo la novela Caesar's Column: A Story of the Twentieth Century de Ignatius L. Donnelly (1890), autor del Preámbulo del Partido.

Palabras clave: People's Party, populismo, historia de los Estados Unidos, utopía, distopía

Según Nadia Urbinati, "sin una narrativa organizativa y un líder que sostenga su pueblo [y que] sea la verdadera expresión del pueblo en su conjunto, un movimiento populista resulta en todo caso ser mucho más 
que [cuando nace]: un sacrosanto movimiento de protesta y respuesta en contra de una tendencia de la sociedad que traiciona algunos principios democráticos de base y, en particular, de igualdad. El populismo va más allá de la retórica populista y de la protesta política. La distinción entre forma de movimiento y forma de gobierno resulta entonces esencial para analizar el populismo" (Urbinati, 2013: 139). Siguiendo esta intuición, me he interesado por dos formas narrativas, dos novelas del movimiento, que luego se convertiría en el People's Party, de carácter Populista estadounidense, el cual, si bien nunca llegó a Washington, alcanzó la administración de algunos estados, como Cansas, Idaho - cuyo gobernador Frank Steunenberg fue asesinado -, Dakota del Norte, Nevada - con el Silver Party - y Colorado, dejando una huella no solo de la narración en la base del movimiento, que luego se convirtió en partido, sino que además configuró parcialmente su posición de gobierno.

Arato y Cohen afirman que el populismo es una "ideología débil", porque quiere devolver al pueblo el lugar de "soberano" por el que espera, rescatarlo de la exclusión y del silencio al cual ha sido reducido por parte de las élites usurpadoras y corruptas, devolverle la posibilidad de volver a gobernar (por lo general el imaginario populista encuentra en alguna parte del pasado una época feliz) (Arato \& Cohen, 2017: 285) y que en su forma soft, puede ser una crítica de las ausencias de la democracia realmente existente. Mientras que en su forma hard, reconocida por otros autores (Eichengreen, 2017: 368), está, en cambio, en tensión subversiva con la estructura misma del sistema democrático. Si el pueblo es entendido como una entidad orgánica, auténtica y moralmente superior, que se encarna en un líder (un salvador) que le ofrece unidad y representación, entonces es imposible pensar en alguna otra legítima expresión del pueblo en la sociedad o en el gobierno. Fuera del pueblo solamente está el nopueblo, los enemigos del pueblo. El populismo, identificando la parte (sí mismo) por el todo, niega así el pluralismo político y social, y la separación entre política y sociedad civil, que está en el corazón de la democracia liberal. En este caso, el populismo estadounidense puede reconocerse en la segunda parte de la definición, más no en la primera, porque siempre estuvo ausente el líder fuerte y carismático, que quedó reducido a una figura difusa y políticamente ambigua.

En lo que se refiere a los contenidos ideales, muchos insisten todavía en que la ideología populista queda siempre indeterminada, débil, privada del fuerte claim moral necesario para sostener una vigorosa movilización. Históricamente, entonces, se habrían buscado estos contenidos ideales 
acoplándos a host ideologies más densas, como el nacionalismo y el racismo, el fascismo o el comunismo, según los tiempos y los contextos locales (Testi, 2018). En este sentido el People's Party estadounidense no puede sino señalar el tentativo de unir muchos host distintos, mas no contrastantes y seguramente precursores de tendencias futuras. En este sentido, he investigado sobre las narrativas más importantes y difundidas alrededor del partido, las cuales marcaron sus políticas de gobierno.

Los textos que he elegido para realizar este análisis son dos: Looking Backward: 2000-1887 de Edward Bellamy publicado por Ticknor \& Co. (y luego por Houghton Mifflin) en Boston, en 1888, y Caesar's Colum: A Story of The Twentieth Century escrito por Ignatius Donnelly y publicado por F. J. Shilte \& Co. de Chicago en 1890 (Mchugh, 1978: 58).

Las dos novelas contienen casi todos los temas relativos a las elecciones $\mathrm{y}$ a los issues fundamentales que provienen del ya variante movimiento populista, del cual se apropia el People's Party en el momento de su fundación y que luego vienen nuevamente inmersos en la política estadounidense a través de los hilos que se desmarañan de la exformación política (M. Schneirov, 1994: 102).

Pero no solamente eso, una de las definiciones de "pueblo" populista más en boga todavía hoy, hasta llegar a ocupar el lugar de Occupy Wall Street, ha quedado "esculpida" en las páginas de una de las dos novelas. Donnelly, de hecho, escribe una variante del famoso eslogan del $99 \%$ (Donnelly, 1896: 95), recordado frecuentemente junto al speech de uno de los exponentes más famosos del People's Party, Mary Elizabeth Lease: "Wall Street posee al país. No es más un gobierno del pueblo, para el pueblo y con el pueblo, sino un gobierno todo de Wall Street, para Wall Street y con Wall Street" (Lease, 1951: 159). Estos eslóganes son repetidos aún en nuestros días durante muchos performances liberales y progresistas en todos los Estados Unidos y expresan un mood que recorre continuamente cada página de las dos novelas y las posiciones de los movimientos prePeople's Party. Los temas que aparecen revelados y que, sin embargo, no resultan ser menos ambiguos en los dos textos, están relacionados con las elecciones y la plataforma del People's Party (la construcción misma de la cual Donnelly participa y de la cual es magna pars) (Kleppner, 1979: 284). En esto, son centrales algunas de la cuestiones que luego vendrán a ser típicas del "populismo", como la forma retórica, política e intelectualfuncional incluso en el siglo XXI: la primera de todas, la contraposición (y la lucha) a las élites (o presuntas élites) por parte del "pueblo" (entendido regularmente, pero de modo confuso, como "nación"), la contraposición 
entre las "periferias" rurales respecto de la administración centralizada (y central), la atención al "mundo femenino" y los disturbios en el sistema comercial y financiero, hijo del capitalismo moderno.

Los dos libros difieren acerca de un argumento: la fe en la bondad de los avances tecnológicos y científicos, así como, sobre todo, en sus recaídas (en las visionarias novelas aparecen: badge, televisores de plasma, teléfonos, etc.), a tal punto que el trabajo de Donnelly viene concebido como un cuento distópico, mientras el de Bellamy, como utópico.

Ambos imaginan el mundo, de modo particular los Estados Unidos del tardío siglo XX y del año 2000. Ambos prefiguran (o prevén) desarrollos tecnológicos que existen y sobretodo contribuyen (positiva o negativamente) a movilizar conciudadanos (y no solamente) hacia el mundo ideal imaginado por un partido que ellos mismos cofundan (Donnelly) o contribuyen a hacer grande (Bellamy).

\section{El círculo de lectores}

Para recordar cuál fue el impacto de estas dos obras sobre la sociedad estadounidense de la época, pero no solamente sobre ella, recordamos que, en la segunda edición, la novela futurista de Bellamy encontró un enorme éxito (Brass, 1996: 194), con más de 400.000 copias vendidas en los Estados Unidos (Schneirow, 2019: 102). De hecho, las ventas superaron los 532.000 volúmenes en los Estados Unidos antes de la mitad del 1939, mientras que según otras fuentes fueron, en realidad, más de un millón (Sreenivasan, 2008: 33). En los años noventa en el Medio Oeste, West and South lanzó miles de ediciones "populares" del libro que fueron vendidas incluso a 5 céntimos de dólar (Robertson, 2018: 70). El libro ganó también un vasto número de lectores en Gran Bretaña, con más de 235.000 copias vendidas entre la primera edición de 1890 y la de 1935.

Pero no solo esto: el club "nacionalista", fundado con el fin de hacer real la novela utópica, se convirtió en poco tiempo en una red organizada de grupos políticos (muchas veces definidos "socialistas"), que emergieron a finales de los años ochenta en toda la Nación. Por lo menos 165 clubes nacionalistas fueron formados por los conocidos como "Bellamytes", que buscaron imaginar (y remodelar de algún modo) la economía y la sociedad, principalmente a través de la nacionalización de la industria. Uno de los últimos números de The Nationalist (su periódico de referencia) contaba con cerca de 500 miembros. A causa del crecimiento del movimiento populista, que en parte hizo suyas las expectativas, los ideales y las 
dificultades tanto financieras como físicas padecidas por Bellamy, los clubes nacionalistas bellamytas empiezan a dispersarse en 1892, pierden su revista nacional en 1894 y para 1896 desaparecen completamente de la escena, con el cierre de la experiencia populista. El mismo Eugene Debs, futuro líder de los sindicatos y del socialismo americano, y en aquellos años motor de las luchas contra Pullman, admite haber vivido un cambio ideológico y político leyendo las páginas de Bellamy (Zinn, 2005: 194).

También Caesar's Column se reveló como un éxito popular, vendiendo 60.000 copias en su primera publicación. Finalmente, sus ventas comprendieron la suma de 250.000 libros. La novela de Donnelly fue un elemento de la gran oleada de literatura utópica y distópica durante el tardío siglo XIX y el inicio del XX, ejemplificado por obras como The Iron Heel de Jack London, autor ligado a la experiencia del variado movimiento prepopulista, luego de publicar Two Thousand Stiffs, un cuento acerca de una de las experiencias relacionadas a las Kelly's (y Coxey's) Army del 1893.

El resultado de esta efervescencia editorial fue una verdadera "batalla de los libros", que duró el tiempo restante del siglo XIX y se extendió hasta los inicios del XX. La naturaleza del debate queda ilustrada en el título de 1891 que Ludwig Geissler diera a su obra Looking Beyond, pensado, entre otras cosas como competencia de Looking Forward de Richard Michaelis ${ }^{1}$.

Sus fortunas traspasaron las barreras nacionales y de la lengua y, sea Donnelly que Bellamy fueron traducidos a muchos idiomas, en algunos casos de carácter nacional verdaderamente reveladores. El trabajo de Bellamy fue traducido al búlgaro en 1892 y en 1900 Bellamy aprobó personalmente una petición del escritor Illiya Yovchev para realizar una "traducción adaptada" basada en el orden social búlgaro. La obra que resultó de ello se tituló El presente visto por nuestros descendientes y una mirada al Progeso del futuro, la cual siguió la misma trama de Looking Backward, con la diferencia de que los eventos, en la versión de Yovchev, se desarrollan en una Sofía respetuosa con el ambiente y describe el recorrido único seguido por el país con el fin de adaptarse al nuevo orden social. Esta obra es considerada por los críticos locales como el primer trabajo utópico búlgaro ${ }^{2}$. El libro de Bellamy tuvo una acogida particularmente "calurosa" en la Alemania guillermina, y comprendió varias parodias

1 Michaelis (1890). Looking Further Forward: An Answer to Looking Backward by Edward Bellamy. Chicago, Illinois: Rand McNally.

2 Рунев (2012). Началото. In Дочка Русева, Стоянка Полонова, Иван Серафимов (ed.), Фантастика-1“. Сборник за научна фантастика и прогностика (pp. 1-16). 
(desde Eduard Loewenthal, Ernst Müller y Philipp Wasserburg hasta Konrad Wilbrand y el citado Michaelis). El libro llegó también a la China, donde fue readaptado por un editor local que lo imprimió casi durante cinco décadas (correspondientes más o menos a la difusión de las ideas y, luego, a la hegemonía del gobierno nacionalsocialista) ${ }^{3}$.

Más allá de la esfera meramente literaria, las descripciones de Bellamy de la planificación urbanística utópica han tenido una influencia práctica sobre la fundación, por parte de Ebenezer Howard, del movimiento de la ciudad jardín en Inglaterra y sobre el proyecto del Bradbury Building en Los Ángeles.

Por su parte el volumen de Donnelly4, encontró el interés de los lectores no norteamericanos, sobretodo en el Norte de Europa contando con traducciones en noruego y en sueco prácticamente en conexión directa, como el más famoso Atlantis del mismo Donnelly ${ }^{5}$ que encantó incluso al mismo Gladstone (Adams, 2005: 46).

Los dos libros, sin duda, han plasmado los años siguientes su exordio no solo por las (casi siempre) felices y futuristas intuiciones científicas y tecnológicas (sobre las cuales mucho se ha escrito), sino también porque han señalado el horizonte de expectativas de muchos (e)lectores, proponiendo algunos temas, que todavía tienen eco en los movimientos y partidos populistas contemporáneos (Lee, 1987: 53).

El mundo anglófono, en todo caso, adoró a Donnelly en particular, y los australianos fueron y se cuentan todavía entre sus más agudos lectores. Para ellos, en un momento de gran crisis económica, Donnelly aparece como un profeta creíble en tiempos de ruina. Ayudó a erigir el aura literaria que llevó al movimiento obrero australiano al debut, a tal punto que el periódico The Bullettin identificaba acertadamente a Donnelly como uno de los principales autores de referencia: muchos sindicalistas eran, o deseaban ser, también pequeños agricultores. Henry Lawson escribió un cuento en el cual un agricultor "descuidado" en el Nuevo Gales del Sur occidental busca mantener el ritmo con "todas las grandes cuestiones sociales y políticas del día” leyendo a Donnelly (Lawson, 2013).

3 Andolfatto (2015). Productive distortions: On the translated imaginaries and misplaced identities of the late Qing utopian novel. In Transtext(e)s Transcultures 跨文本跨 文化, 10 [13.07.2018].

${ }^{4}$ Cf. Abbott (2010). Political Thought in America: Conversations and Debates, Fourth Edition. Long Grove: Waveland Press, p. 187.

5 Abbott (2017). Master of Disaster, Ignatius Donnelly. In The Public Domain Review, 27 September 2017, [12.11.2018]. 
Algunos polemistas contemporáneos culpan, de hecho, al escritor de la decadencia de la izquierda australiana: "Cuando el partido de los trabajadores emerge con fuerza política, la penetrante influencia de Donnelly, contribuyó a consolidar su instintiva convicción que su verdadero enemigo en Australia no fuera el capitalismo en general, como una banda clandestina de bolsistas y banqueros ('el poder del dinero') que inclinaba la balanza hacia la explotación de los productos, las pequeñas empresas y los agricultores, así como a las fuerza de trabajo. Esta mentalidad populista, junto a otro tipo de influencias no marxistas [...], han contribuido a garantizar que desde el inicio el Labour no haya perseguido nunca una visión global del socialismo basada en ideas todavía no diluidas de la lucha de clases" (Holt, 2018).

\section{Los temas}

\subsection{La lucha contra las élites: narraciones políticas}

En el centro de las dos novelas se encuentra la lucha contra las élites (una afortunada y una desafortunada) y solo en algunos pasajes, estas élites vienen representadas por las big corporations. Esta lectura aparece como coherente con las políticas expresadas por parte de los gobiernos (nacionales) populistas que señalaron a las élites de las constructoras de infraestructuras (sobre todo de ferrovías), de los funcionarios públicos, pero sobretodo y en último grado de los "políticos de profesión". En 1892 cuando en Colorado se estableció un tique populista, por ejemplo, las elecciones de la nueva administración marcaron los temas denominados como "clásicos" para los populistas de cada siglo: "the regulation of state railroad rates, the free coinage of silver at sixteen to one, the eight-hour day and the reduction of salaries of state officials" (McCarthy, 1973: 146-155). La única cuota fue también una indicación en parte sugerida por Bellamy y George. Mientras que ahora hay muchos estudios que dan cuenta de cómo fueron fuertemente penalizados los "funcionarios públicos", como ha sido indicado en la plataforma del People's Party de Kansas (1900).

Seguramente también y, sobre todo, la plutocracia (Bellamy - "The real issue is 'between men and money', in Bellamy's phrase; and they cannot afford to side with money against men") (Demarest Lloyd, 1896: 281) u oligarquía (Donelly) es la clase por combatir; la batalla prevé la puesta a un lado de la clase de los banqueros (que han de ser superados, según los dos autores). 
Un enemigo imprescindible son los intelectuales. Un hombre como William Jennings Bryan, un líder democrático muchas veces candidato a la presidencia, también en nombre del People's Party, podía denunciar a los intelectuales como una élite arrogante, una "little irresponsible oligarchy of self-styled "intellectuals", un "scientific soviet" (Bloom, 2015: 78) (incluso después de 1919), que cultivaba las frías razones de la ciencia en contra de aquellas calurosas "populares" del corazón. Bryan podía decir: "Mind worship is the great sin in the intellectual world today" (Testi, 1984, 37).

La nueva élite populista es, entonces, para los dos autores examinados, algo disímil de aquella contrastada realmente por parte de quienes han gobernado los distintos estados de carácter populista ${ }^{6}$ (prueba de ello es la empresa de Frank Steunenberg - a tal punto que junto al monumento a él dedicado se encuentra escrito: "Law and order?"), así como la selección de la clase dirigente imaginada como una mixtura entre ciudadanos elegidos a través del sufragio y el fruto de la división del trabajo de tipo corporativo: "He is the President of the United States [...] or rather the most important function of the Presidency is the headship of the industrial army" (Bellamy, 1890: 53); "Do you know the method of electing officials by votes of the retired members of the guilds is nothing more than application on a national scale of the plan of government by alumni, which we used to slight extent occasionally in the management of our higher educational institutions" (Official Proceedings of the Democratic National Convention Held in Chicago, 1896, 5).

Una parte interesante y actual del programa de Bellamy previó también que el derecho (su profesión inicial, así como la de Donnelly), se convirtiera en una ciencia obsoleta y que también todos los funcionarios a ella relacionados desaparecieran (Bellamy, 1890: 58-59). Entre otras cosas, Bellamy preveía que ningún trabajador fuese un "ignorante", sino que el sistema de escolarización alcanzara todos los grupos/clases/estratos sociales (Bellamy, 1890: 63). De acuerdo con Bellamy, la nueva sociedad habría debido fundarse "on the true self-interest of a rational selfishness, and appealing to the social and generous instincts of men" (Bellamy, 1890: 77).

Cuán potentes e influyentes fueron en verdad las visiones antielitistas expresadas (más no suscitadas) por parte de los trabajos de Bellamy y Donnelly, es descifrable a través de la retórica de los futuros representantes

6 Weir (2013). Workers in America: A Historical Encyclopedia, Vol. 1, p. 330.

7 Statue of Past-Governor Frank Steunenberg on the Grounds of the State Capitol Building, Boise, Idaho. In Smithsonian Art Inventory Sculptures, [07.10.2018]. 
del partido y, luego, de la historia americana en general. Casi al final de su novela utópica, Bellamy escribe, por ejemplo: "I have been in Golgotha [...] I have seen Humanity hanging on a cross!!” (Bellamy, 1890, 87). La cruz de la pobreza. Y continúa: "Better your part pleading for crucified humanity with a scoffing generation, than here, drinking of wells you digged not, and eating of trees whose husbandmen you stoned". Estos reclamos de aquel entonces, estarán presentes en la retórica americana posterior. Baste recordar que Bryan en 1896, en la Convención democrática, viene nombrado candidato a la presidencia proclamado como: "You shall not crucify mankind upon a cross of gold8"; donde esta última afirmación comprendía de nuevo la lucha por el bimetalismo.

El lenguaje populista parece jugar un rol importante en la política estadounidense, no solo a través de Bryan, candidato democrático y populista ("Las personas sufren hasta que el sufrimiento deja de ser una virtud; son pacientes hasta que la paciencia los desborda y, entonces, si despiertan, pierden las riendas del gobierno y vuelven a ponerlo sobre su base original [el pueblo mismo]") (Magliocca, 2006: 821). En los años treinta también Franklin Roosevelt apela al "forgotten man at the bottom of the economic pyramid" (Humes, 2008: 152), dándole la vuelta al sentido dado al mismo tiempo por un darwinista social; y para 1992 Bill Clinton se lanza a la presidencia tomando prestado el lema "put people first" y "invest in people" (Dover, 2006: 74), elementos de corte populista que llegarán también a Europa, gracias, por ejemplo, al "pensamiento disidente" sobre la política monetaria de Ezra Pound, cuya historia familiar se entrecruza con la lucha a favor del bimetalismo, que es el otro caballo de batalla del populismo americano (Dover, 2006: 74).

Hermet escribe que el People's Party, en el fondo, llegó a combinar dos propuestas: la "propuesta política 'plebeya' contra los ricos, exaltando la lealtad de los agricultores del Medio Oeste o mineros del oeste hacia la visión antiurbana y agraria, y un populismo étnico (en el marco de sus prejuicios protestantes del norte y de sus secuaces que se habían revelado como abiertamente racistas, de acuerdo con el politólogo)" (Hermet, 2001: 121), al mismo tiempo que toca otro tema importante de la revolución contra las élites: el soterrado racismo del cual también los libros de Bellamy y Donnelly pueden llegar a ser calificados. En esto, sin embargo, los dos autores son abiertamente "nacionalistas" y populistas (en el sentido en que

$8 \mathrm{http} / /$ www.milestonedocuments.com/documents/view/william-jennings-bryanscross-of-gold-speech [19.12.2018]. 
los movimientos de estos en el Sur del país habían agregado también formas de protesta de los afroamericanos): su desprecio apunta sobre todo hacia los hebreos (en cuanto "globalizadores/transnacionales" y especuladores) y sus nuevos inmigrantes estadounidenses (entonces italianos, ciudadanos de países de Europa del este y chinos) (Stromquist, 2006). Tanto es así que la primera cosa que impacta, leyendo la novela de Donnelly, es el hecho de que el protagonista provenga del católico Cantón Uri, donde aún en el 2018, una corporación posee cerca del 70\% de la superficie del cantón (principalmente prados alpinos y bosques), entendido claramente como un ejemplo de buena democracia "imaginada".

\subsection{La Nación, la democracia y el mundo}

El pueblo al cual se dirigen estos autores es la nación: así como hizo el People’s Party, más allá de su parábola peculiar y del diálogo con la patrulla de nacionalistas americanos ("People will do well to remember that the People's Party is not an ideal organization from the standpoint of Nationalism. It is a reform body with its face set against trusts, monopolies, and Wall Street, and as such commands respect ${ }^{10}$ "). Un elemento interesante y que hasta hace unos pocos años no parecía como contemporáneo era esa visión completamente cerrada de la nación del año 2000 propuesta tanto por Bellamy, como por Donnelly. De hecho, mientras Donnelly propone paradójicamente dos tipos de nacionalismo: uno malvado - los USA - y uno bueno - la Federación africana, Bellamy se imagina solo un nacionalismo que alcanza su ápice (óptimo) en la apertura al intercambio económico igualitario ("a vast industrial partnership") (Bellamy, 1888: 37), en el cual, del comercio nace una paz en el que todas las naciones son autónomas (Bellamy, 1888, 39) y las migraciones son rígidamente controladas (baste la cita de las "vile hordes of Mongolian coolies") (Bellamy, 1888: 39).

El poder para las "pobres criaturas humanas" (Donnelly, 1890: 81) se construye dentro de los confines nacionales contra la economía de la oligarquía (de origen hebrea) cosmopolita. En algunos casos, la mejor de las soluciones previstas es aquella de la vuelta a partir de los territorios colonizados (por ejemplo, la imaginaria - immaginifica - Uganda).

9 Siegenthaler (2018). Dove la democrazia diretta è praticata nella sua forma più autentica. In Swissinfo.ch, 30 de julio del 2018, [07.10.2018].

10 Franklin (1938). Edward Bellamy and the Nationalist Movement. In The New England Quarterly, Vol. 11, n. 4, p. 766. 
Ningún transnacionalismo queda previsto en estas novelas (ni mucho menos internacionalismo), no es bienvenido el multiculturalismo, pero está prevista la movilización de las fuerzas de trabajo potentemente regimentadas por los gobiernos de los estados (Lye, 2005: 63). Y esto vale tanto para Bellamy, concebido, incluso como filosocialista (en realidad corporativista), como para Donnelly, quien prevé no solo un cierre nacionalista (confederado al máximo), sino también una jerarquización interna "meritocrática" muy fuerte, mayor que en Bellamy, para quien el poder del Gobierno (es decir, de la rama ejecutiva del Estado-Nación) es casi inaferrable y solo es posible una "machine to insure justice and help people” (Donnelly, 1890: 89).

En ambos, la primera "nación" puesta en la mira es aquella del pueblo hebraico ("The aristocracy of the world is almost altogether of Hebrew origin" expresado en el pasaje "The world is to-day Semitized") (Donnelly, 1890: 24; 82) y aquella del nuevo Estado-Nación italiano (lleno de anarquistas y llevado a acumular riquezas vendiendo presuntos títulos nobiliarios) (Donnelly, 1890: 52). A estos se contraponen débilmente algunos miembros de la antigua aristocracia alemana bien instruidos, pero ahora caídos en desgracia (Maximilian Rudolph en Caesar's Column).

A esta visión y a la lectura del People's Party, subtiende también magníficamente otra obra (la más conocida) de Donnelly sobre la Atlántida: no ha sido negado un origen común de la humanidad, sino que queda relegado a un pasado remotísimo y mejor que el presente de aquel que conoceremos. No se trata de una prueba de la Golden age, sino de leyendas y cuentos (aunque platónicos); ni mucho menos de una memoria (obviamente), como un quedarse anclado en una nostalgia pesimista y melancólica premoderna (Beringer, 2012). Es este símil, del África idílica imaginada en el último capítulo de Caesar's Column, donde no solo se habla de salario fijo y del fin de la relación entre moneda y oro, sino también del fin de la especulación financiera y de la importancia de ser felices sin necesidad de ser millonarios, sino criadores de "our little world", como reclama el imaginado origen del sueño del West (Donnelly, 1890, 265).

A todo esto, se une la profunda lejanía de la idea de la democracia liberal: "The rich men owned the newspaper and the newspaper owned their readers; so that, practically, the rich men cast all those hundreds of thousands of votes" (Donnelly, 1890: 139), mientras que para Bellamy, la nación es sobre todo "one great corporation" (Bellamy, 1888: 15). Bien sea porque ha sido infestada por las "maquinarias partidistas", bien sea 
porque una vez superada por una forma de "democracia", concebida de un nuevo modo a la altura de los tiempos: como el corporativismo y el reconocimiento de la "meritocracia" (cooptación al interior del sistema nacional). Para Bellamy el Estado del futuro aplicará, contemporáneamente, la idea del ciudadano-productor, y aquella del ciudadano-consumidor, a través de una especie de palanca "industrial", semejante a la militar, pero aplicada a los dos sexos. Y mientras Bellamy augura que "the goods are the nation's" (Bellamy, 1888: 28), Donnelly vaticina "our governing body, called The People", dividido en tres ramas (productores, manufactores y los "literatos") (Donnelly, 1890: 253).

En estos aspectos el 2000 de los dos escritores en verdad asemeja al futuro (para ellos a casi medio siglo de distancia) de los nuevos Estadosnación: China, Italia y Portugal de los años veinte y treinta ${ }^{11}$.

\section{Bibliografía}

Adams, M. (2005). Meet Me in Atlantis: My Obsessive Quest to Find the Sunken City. New York: Penguin.

Arato, A. \& Cohen, J. (2017). Civil Society, Populism and Religion. Constellations, 24, pp. 283-295.

Bellamy, E. (1888). Looking Backward: 2000-1887. Boston: Ticknor \& Co.

Beringer, A. (2012). 'Some Unsuspected Author': Ignatius Donnelly and the Nineteenth-Century American Conspiracy Novel. Arizona Quarterly, 68, Winter, pp. 35-59.

Bloom, P. (2015). Fracture: Life and Culture in the West, 1918-1938. New York: Basic Book.

Brass, T. (1996). Popular culture, populist fiction(s): The Agrarian Utopia of A. V. Chayanov, Ignatius Donnelly and Frank Capra. The fournal of Peasant Studies, 24, pp. 153-190.

Demarest Lloyd, H. (1896). The Populists at St. Louis. Review of Reviews, XIV, September, pp. 278-283.

Donnelly, I. (1890). Caesar's Column: A Story of the Twentieth Century, Caesar's Column: A Story of the Twentieth Century. Chicago: F. J. Shulte \& Co.

Dover, E. D. (2006). Images, Issues, and Attacks. Television Advertising by Incumbents and Challengers in Presidential Elections. Lanham: Lexington Books.

Castleton, E. (2012). Une 'armèe d'hérétiques' face à une 'croix d'or'. Le premier populisme américain et l'hétérodoxie monétaire. Critique. Revue générale des publications françaises et étrangères", 776-777, pp. 28-37.

11 Este tema se compararía con la tesis sobre "cronología populista" en el libro de F. Finchelstein (2017). From Fascism to Populism in History. Buenos Aires: Press of University of California. 
Eichengreen, B. (2018). Populism, Ideology and Materialism. New Global Studies, 12/3, pp. 367-375.

Finchelstein, F. (2017). From Fascism to Populism in History. Oakland: University of California. [Spanish translation: Del fascismo al populismo en la historia (riv. 2018). Buenos Aires-Mexico-Madrid-Bogotá: Taurus, University of California Press.]

Frank Leslie's popular monthly (1893), 35, pp. 203-205.

Hermet G. (2001). Les populismes dans le monde. Paris: Fayard.

Holt, S. (2001). A prophet of globalisation: Ignatius Donnelly. On Line Opinion, 15 September 2001.

Humes, J. C. (2008). The Wit \& Wisdom of FDR. New York: Harper Collins.

Kleppner, P. (1979). The Third Electoral System, 1853-1892: Parties, Voters, and Political Cultures. The University of North Carolina Press.

Lawson, H. (2015). While the Billy Boils: The Original Newspaper Versions. Sydney: Sidney University Press.

Lease, M. E. (1981), Speech to the Woman's Christian Temperance Union. In Jensen, J. M., With These Hands: Women Working on the Land, pp. 154-160. New York: The Feminist Press and McGraw-Hill Book Company.

Lee, B. (1987). American Fiction 1865-1940. New York: Routledge.

Lye, C. (2005). America's Asia: Racial Form and American Literature, 1893-1945. Princeton: Princeton University Press.

Magliocca, G. N. (2006). Constitutional False Positives and the Populist Moment. Notre Dame Law Review, March, pp. 821-888.

McCarthy, G. M. (1973). The People's Party in Colorado: A Profile of Populist Leadership. Agricultural History, Vol. 47/2, pp. 146-155.

Mchugh, C. (1978). Midwestern populist leadership and Edward Bellamy. "Looking backward" into the future. American Studies, 19/2, pp. 57-74.

Mudde, C. (2015). Populism in Europe and the Americas: Threat or Corrective for Democracy? Cambridge: Cambridge University Press.

Official Proceedings of the Democratic National Convention Held in Chicago, Illinois, Fuly 7, 8, 9, 10, and 11, 1896, Logansport, Indiana, 1896, 226-234. Reprinted in The Annals of America, Vol. 12, 1895-1904: Populism, Imperialism, and Reform (1968), pp. 100-105. Chicago: Encyclopedia Britannica, Inc.

Pfaelzer, M. J. (1974). Utopian Fiction in America. Pittsburgh: University of Pittsburgh Press.

Postel, C. (2008). The populist Vision. Oxford: Oxford University Press.

Robertson, M. (2018). The Last Utopians: Four Late Nineteenth-Century Visionaries and Their Legacy. Princeton: Princeton University Press.

Schneirow, R. (2019). Uncovering the contradictions in Samuel Gosper's "More": reading "What does Labor want? The fournal of Gilded Age and Progressive Era, 18, pp. 99-119. 
Stromquist, S. (2006). Re-inventing 'The People': the Progressive Movement, the Class Problem, and the Origins of Modern Liberalism. Urbana and Chicago: University of Illinois Press.

Tarchi, M. (2018). Italia populista. Bologna: il Mulino.

Testi, A. (1984). L'età progressista negli Stati Uniti. Bologna: il Mulino.

Testi, A. (2018). Appunti. Populismo e religione negli Stati Uniti. Short Cuts, 3 April 2018.

Twenton, D. J. (1993). Considering Why Populism Succeeded in South Dakota and Failed in North Dakota. South Dakota State Historical Society, 7, pp. 330-344. Urbinati, N. (2013). The Populist Phenomenon. Raisons Politiques, 51, pp. 137-154. Zinn, E. (2005). Storia del popolo americano. Milano: Il Saggiatore. 